\title{
A multi-enhancer RET regulatory code is disrupted in Hirschsprung disease
}

Sumantra Chatterjee,,${ }^{1,+}$ Kameko M Karasaki, ${ }^{2}$ Lauren E Fries ${ }^{1}$, Ashish Kapoor ${ }^{3}$ and Aravinda Chakravarti ${ }^{1,+}$

${ }^{1}$ Center for Human Genetics and Genomics, New York University Grossman School of Medicine, New York, NY 10016; ${ }^{2}$ Department of Biology, Johns Hopkins University, Baltimore, MD 21205, USA; ${ }^{I}$ nstitute of Molecular Medicine, McGovern Medical School, University of Texas Health Science Center at Houston, Houston, TX 77030, USA.

+Send all correspondence to:

Aravinda Chakravarti, Ph.D.

Center for Human Genetics \& Genomics

New York University Grossman School of Medicine 435 East $30^{\text {th }}$ Street, Room 802/3

New York, NY 10016

T: (212) 263-8023

E: aravinda.chakravarti@nyulangone.org

Sumantra Chatterjee, Ph.D.

Center for Human Genetics \& Genomics

New York University Grossman School of Medicine

435 East $30^{\text {th }}$ Street, Room 823R

New York, NY 10016

T: (212) 263-8029

E: sumantra.chatterjee@nyulangone.org 


\section{Abstract}

The major genetic risk factors for Hirschsprung disease (HSCR) are three common polymorphisms within cis regulatory elements (CREs) of the RET receptor tyrosine kinase gene that reduce its gene expression during enteric nervous system (ENS) development. These variants have synergistic effects on RET gene expression and additionally dysregulate other ENS and HSCR genes in the RET-EDNRB gene regulatory network (GRN). Here, we use siRNA, ChIP and CRISPR/Cas9 deletion analyses in the SK-N-SH cell line to ask, how many HSCR-associated risk variants reside in CREs and affect RET gene expression? We demonstrate that $31 \mathrm{HSCR}$-associated variants reside in candidate RET CREs, ten with differential allele-specific in vitro enhancer activity and seven affecting RET gene expression; of these, five bind the transcription factors PAX3, RARB, GATA2 and SOX10. These and our prior results demonstrate that common sequence variants in at least 10 RET enhancers affect HSCR risk, extending the known RET-EDNRB GRN to reveal an extensive regulatory code modulating disease risk even at a single gene. 


\section{Introduction}

It is now well established that most human complex traits and diseases arise from the additive genetic effects of hundreds to thousands of variants distributed across the genome $^{1}$. At each locus, multiple statistically significant variants are detected but it is unknown how many of them make functionally independent contributions to the phenotype. The widespread existence of genetic association (linkage disequilibrium, LD) between local sequence variants makes this a difficult question to answer by statistical methods alone, and requires experimental perturbation and assessment of each candidate variant ${ }^{2}$. This is because genetic associations between variants depend on their recombination frequency, not their functional effects, thereby associating causal with innocent variants.

The majority of causal variants that contribute to trait variation reside within cis regulatory elements (CRE) and enhancers of a target gene thereby modulating its gene expression, usually in a cell-type specific manner ${ }^{3,4}$. Such gene expression control is assumed to occur within a topologically associating domain (TAD) ${ }^{5,6}$, defining the physical locus within which CREs function. However, three major questions remain unanswered. First, since a TAD usually harbors multiple CREs and genes, which CREs affect which gene's expression? Second, do the different CREs of a specific gene have unique functions in space, time and cellular states or are they redundant (shadow enhancers)? Do they act independently, or are they synergistic and require clustering (super enhancers) for function 3,7,8? Third, since most CRE effects are small how do such small gene expression effects modulate phenotypes? The existence of many experimental methods to identify CREs comprehensively now allows us to address these questions ${ }^{9,10}$.

In this study, we use Hirschsprung disease (HSCR; congenital colonic aganglionosis) as an exemplar to identify the number and activity of all ENS enhancers with diseaseassociated variants at its major gene RET. HSCR is a complex neurodevelopmental disorder in which failure of differentiation of enteric neural crest cell (ENCC) precursors during ENS development leads to aganglionosis; >35 genes/loci explaining $67 \%$ of its population attributable risk have been identified ${ }^{11}$. Significantly, most of this risk arises from coding and enhancer variants of the RET receptor tyrosine kinase gene with smaller 
contributions from other genes, all of whose functions in ENS development are united through a gene regulatory network (GRN) that co-regulates $R E T$ and EDNRB ${ }^{2,11-13}$. Thus, we also examine whether the CRE sequence variants we identify are individually sufficient to perturb RET gene expression as well as GRN activity.

Specifically, using the human neuroblastoma cell line SK-N-SH, we test 31 HSCRassociated common (minor allele frequency, MAF $\geq 10 \%$ ) polymorphisms at the RET locus ${ }^{14}$ to demonstrate that 22 lie within CREs and of which 7 have sequence variants with differential enhancer and RET gene expression activity; in addition, 3 were identified before 2. Two of the new CREs bind the transcription factor (TF) PAX3, known to affect neural crest cell migration and ENS differentiation ${ }^{15,16}$. Reducing PAX3 expression decreases RET gene expression as well as that of SOX10, the major TF in the RET-EDNRB GRN 2,17,18. Deleting individual RET CREs using CRISPR/Cas9 genome editing reduces RET expression but is insufficient to perturb genes expression for other GRN genes. We know that RET-EDNRB GRN effects are evident only when RET gene expression falls below $50 \%$ of its wildtype level ${ }^{12}$. Therefore, in HSCR, significant reductions of gene expression at RET and its GRN to affect ENS development only occurs in individuals with multiple CRE variants in combination with variants in other GRN genes. The corollary is that a diverse regulatory code affects complex disease genes.

\section{$\underline{\text { Results }}$}

Enhancers at the RET locus

To create a complete catalog of common (MAF $\geq 10 \%) R E T$ regulatory variants associated with HSCR, we began with analysis of all 38 genome-wide significant non-coding single nucleotide polymorphisms (SNPs) discovered in a genome-wide association study (GWAS) of 220 HSCR trios comprising a proband and both of her/his parents ${ }^{14}$. These SNPs were distributed across 6 LD blocks in a 155kb TAD (chr10: 43434933-43590368; hg19) containing RET as the sole gene (Figure 1A). We previously analyzed 8 of these SNP-containing genomic elements because they each disrupted a predicted TF binding site (TFBS) determined form ENCODE ChIP-seq data ${ }^{2}$. 
We first asked: do the 30 remaining SNPs resided within CREs? We conducted in vitro functional tests of enhancer activity by cloning $\sim 500$ bp elements centered on each risk variant (Table 1) into a pGL4.23 luciferase vector, with a minimal TATA-box of the $\beta$-globin gene and transfecting them into the human neuroblastoma SK-N-SH cell line. SK-N-SH expresses all known members of the RET-EDNRB GRN and is an appropriate cell model system for studies of ENS transcriptional regulation ${ }^{2,12}$. Two pairs of SNPs (rs17158318/ rs17158320 and rs2506021/ rs2435342) were only 64bp and 108bp apart and were cloned into the same elements (E9 and E24, respectively) (Table 1). As positive controls, we reanalyzed 3 HSCR associated SNPs (rs2506030, rs7069590, rs2435357) previously shown to be RET enhancer variants ${ }^{2}$ (Table 1). Our reporter assays showed that 22 new elements had significant enhancer activity $(P<0.001$ and $>2 X$ reporter activity over the promoter only control vector) of which 7 (E2, E4, E5, E14, E26, E27 and E28) also displayed differential reporter activity between the risk and non-risk alleles (Table 1 and Figure 1B). Among the latter, 71\% (E2, E4, E26, E27 and E28) overlapped an open chromatin region or an enhancer-associated epigenetic mark in the human fetal gut and SK-N-SH ${ }^{19,20}$ while $20 \%$ (E9, E16, E21) of the remaining 15 elements with reporter activity that had no allelic difference overlapped a potential enhancer mark (Figure 1A). Thus, at least 10 functionally distinct CREs within the RET TAD can potentially affect HSCR risk.

\section{Haplotype specific effect of causal RET polymorphisms}

The identification of 10 CRE-associated risk variants (rs788263, rs788261, rs788260, rs2506030, rs1547930, rs7069590, rs2435357, rs12247456, rs7393733 and rs2505541;

Table 1) prompted us to ask which allelic combinations were associated with disease risk. First, we estimated haplotypes and their frequencies for all 10 SNPs in 220 unrelated HSCR cases $^{14}$ and 503 unrelated controls from the 1000 Genomes project ${ }^{21}$, all of non-Finnish European-ancestry. Second, we estimated the odds ratio (OR) for all haplotypes with a frequency $\geq 1 \%$ in controls. We observed 10 distinct haplotypes of which CTGAACCACT (risk allele in bold) was used as the reference because it had the smallest number (one) of risk alleles (Table 2) and we have previously shown that HSCR risk scales with increasing number of CRE variants ${ }^{2,11-13}$ : significant risk was observed for two haplotypes, GCAGGTTGGT (OR 12.2, 95\% Cl: 5.97-24.93, P=7.02×10-12) and CTGAGTTGGT (OR 7.2, 95\% Cl: 3.26-15.91, $\mathrm{P}=1.02 \times 10^{-6}$ ) (Table 2). Unsurprisingly, these two haplotypes contain 
our previously identified risk increasing ATT and GTT haplotypes (for rs2506030, rs7069590, rs2435357) ${ }^{2}$. The 10-SNP risk haplotypes differ only for the first four SNPs (rs788263, rs788261, rs788260, rs2506030) which occur within the most 5' LD block. SNPs within this LD block do contribute to HSCR but we do not have a sufficient sample size to test our hypothesis that GCAGGTTGGT (OR 12.2) has a significantly higher risk than CTGAGTTGGT (OR 7.2). Even discounting these variants, HSCR risk is clearly spread over at least three LD blocks suggesting multiple independent enhancer variants contributing to risk (Figure 1). This is prima facie evidence that these SNPs contribute to risk synergistically. In other words, risk of or protection from HSCR depends on the simultaneous binding or lack-of-binding of multiple independent TFs at RET CREs, implying a $R E T$ regulatory code.

\section{Transcription factors regulating RET}

To identify the TFs that underlie this code, we searched for TFBSs within the 7-novel riskassociated CREs and identified four candidate TFs: (1) PAX3 binding to E2 (AATAAACCC; $\mathrm{P}=4.67 \times 10^{-5}$ ) and E27 (TCGTCACTCTTAC; $\mathrm{P}=9.99 \times 10^{-5}$ ), (2) ZBTB6 to E5 (TGGCTCCATCATG; $P=2.387 \times 10^{-6}$ ), and, (3) ZNF263 binding to E14 (GCCTCACTGCTCCAG; $\mathrm{P}=8.09 \times 10^{-5}$ ). To determine their relevance for HSCR, we performed qPCR in SK-N-SH cells and observed no expression for ZNF263 and ZBTB6 (data not shown). Further, their expression was absent in the developing mouse gut ${ }^{22}$, where $R E T$ expression is critical for ENS development ${ }^{23}$, making it highly unlikely that these TFs control RET expression via specific enhancers. Moreover, ZNF263 and ZBTB6 are both zinc finger domaincontaining proteins that are GC-rich, a feature which overestimates their statistical significance owing to their rarity.

In contrast, we detected robust expression of PAX3 in both SK-N-SH and the developing mouse gut ${ }^{22}$. Thus, we performed ChIP-qPCR for PAX3 in SK-N-SH cells and detected significant binding at both E2 (18-fold enrichment; $P=10^{-3}$ ) and at E27 (26-fold enrichment, $P=$ $5 \times 10^{-4}$ ) (Figure 2). We further demonstrated the specificity of this binding by performing ChIPqPCR after siRNA-mediated knockdown of PAX3 in SK-N-SH cells to show a 1.3-fold ( $P=8 \times 10^{-}$ ${ }^{3}$ ) reduced binding at E2 and 2-fold $\left(P=4 \times 10^{-4}\right)$ reduced binding at E27 (Figure 2). To further 
prove that PAX3 does indeed control RET, we also measured RET gene expression after siRNA-mediated knockdown of PAX3. As positive controls, we measured RET levels after siRNA-mediated knockdown of the established RET TFs SOX10, GATA2 and RARB. These experiments showed that decreasing $P A X 3$ led to a $49 \%\left(\mathrm{p}=4 \times 10^{-4}\right)$ reduction in $R E T$ in comparison to $76 \%\left(p=2.3 \times 10^{-6}\right), 50 \%\left(p=3.1 \times 10^{-3}\right)$ and $81 \%\left(p=4.1 \times 10^{-5}\right)$ decreases consequent to SOX10, GATA2 and RARB knockdown, respectively; as a control, knockdown of $R E T$ by its specific siRNA reduced its expression by $96 \%\left(p=4.4 \times 10^{-6}\right)$ (Figure $3 A$ ). We have previously demonstrated that there is considerable cross-talk between the established RET TFs ${ }^{2}$. Consequently, we measured gene expression of SOX10, GATA2 and RARB after siRNA-mediated knockdown of PAX3: we observed only a significant drop in SOX10 gene expression ( $32 \%$ decrease, $\left.\mathrm{P}=3 \times 10^{-3}\right)$; GATA2 and $R A R B$ levels were decreased but not significantly so (Figure 3B).

\section{in vivo evidence for RET enhancers:}

The human genetic evidence for HSCR-associated polymorphisms within CREs identified from in vitro (reporter activity) and ex vivo (siRNA in SK-N-SH) experiments can be buttressed by deletion analysis of each enhancer rather than knockdown of its cognate TF. To do so, we designed a single guide RNA close to each HSCR-associated SNP for all 10 target enhancers to introduce non-homologous end joining-induced deletions in SK-N-SH cells. We screened 5 independently transfected wells for each guide and verified them by Sanger sequencing: none of the deletions were > 10bp. Except for enhancer E5, successful deletions were observed in the remaining CREs encompassing the polymorphic site. We used the Inference of CRISPR Edits (ICE) tool ${ }^{24}$ to estimate that individual guides introduced deletions $>3 \mathrm{bp}$ in $10-50 \%$ of the cells in all successfully targeted CREs (Supplementary Table 1).

We next measured RET gene expression in these enhancer-deleted cells. Our results demonstrate that except for enhancers E2 and E14, deletion of DNA sequences surrounding the HSCR-associated SNPs in all other CREs led to changes in RET expression. Thus, deletion of E4 (24\%; $\left.p=3.2 \times 10^{-4}\right)$ leads to higher expression while deletion of E26 $(28 \% ; p=$ 3.7x10-4), E27 (19\%; $\left.p=1.2 \times 10^{-3}\right)$ and E28 $\left(29 \% ; p=3.2 \times 10^{-4}\right)$ all lead to lower RET expression (Figure 4A). The positive controls, RET-7 $\left(22 \% ; p=1.3 \times 10^{-3}\right)$, RET-5.5 $\left(22 \% ; p=2 \times 10^{-3}\right)$, and 
RET+3 $\left(32 \% ; \mathrm{p}=4.1 \times 10^{-4}\right)$ reduced $R E T$ gene expression as expected. It is interesting that all 4 intronic enhancers (RET+3, E26, E27 and E28) residing within a $8.5 \mathrm{~kb}$ region (chr10:43,581,812-43,590,347) in the first intron of RET independently control RET expression. Thus, these elements might be a part of a single enhanceosome critical for spatiotemporal expression of RET; note that the risk-associated variants at these sites are on a single haplotype (Figure 1A).

HSCR is associated with loss of RET function ${ }^{25,26}$ and diminished RET expression leads to loss of ENS during gut development in mice, the hallmark of HSCR ${ }^{22,27}$. Thus, we predict that the cumulative effect of the disruption of all HSCR-associated CRE at E4, RET-7, RET5.5, RET+3, E26, E27 and E28 should lead to reduced RET expression. Normalized RET expression averaged over individual enhancer deletion experiments indeed demonstrates that RET expression is diminished by $16 \%(\mathrm{p}=0.003)$ when compared to CAS9 only transfected (wildtype) cells (Figure 4B). Thus, irrespective of the direction of change in RET expression due to individual deletion, the cumulative disruption of these enhancers leads to RET deficiency.

We next asked whether these changes in RET expression had concomitant changes in the expression of the remaining members of the RET GRN by quantifying expression of EDNRB, SOX10, GATA2, RARB, NKX2.5 and PAX3, the members of the GRN which expresses in this cell line, in the individual enhancer-deleted cells. Our results demonstrate no significant changes in gene expression (Figure 4C). This result is not unexpected given our previous finding that GRN transcriptional dysregulation occurs only when RET gene expression falls below $50 \%$ of wildtype levels ${ }^{2,12}$.

Since the individually strongest HSCR associations were within the previously discovered RET-7, RET-5.5 and RET+3 enhancers (OR of 1.8, 1.7 and 4.1 respectively) ${ }^{2}$, we wanted to ascertain if DNA sequences beyond the polymorphic site impacted RET expression. Thus, we generated larger deletions in these enhancers using paired guide RNAs to direct espCAS9 to these specific DNA sequences in SK-N-SH (Figure 4D). We screened 48 independently transfected wells for each enhancer and detected only heterozygous deletions for each. For 
RET-7, we identified 2 clones (4\%) with 169bp and 183bp deletions, both of which deleted rs2506030. We detected 3 heterozygous clones for RET-5.5 deletion (6.25\%): 2 clones with 147bp and 1 clone with a 139bp, all of which deleted rs7069590. Similarly, we also identified 3 clones $(6.25 \%)$ with RET+3 deletion with 1 clone encompassing $147 \mathrm{bp}$ and 2 clones encompassing 138bp deletions, all of which deleted rs2435357(Figure 4D). Next, we measured gene expression of RET, EDNRB, SOX10, GATA2, RARB, NKX2.5 and PAX3 by qPCR in these cells to detect the effect of heterozygous enhancer deletions on the expressed genes of the RET GRN. Deletion of RET+3 led to $32 \%\left(\mathrm{P}=3.1 \times 10^{-3}\right)$ loss of $R E T$ expression compared to cells transfected with only a Cas9 vector without the guide RNA; the corresponding decreases for RET-5.5 and RET-7 were 22\% $\left(P=2.8 \times 10^{-3}\right)$ and 20\% $\left(P=2.6 \times 10^{-}\right.$ ${ }^{3}$ ), respectively, consistent with their individual risks (OR of 4.1, 1.7 and 1.8, respectively) (Figure 4E) (Chatterjee et al., 2016; Emison et al., 2010; Kapoor et al., 2015). Thus, deletion of sequences beyond the region immediately surrounding the polymorphisms do not seem to have any substantial effect on RET expression. Thus, for HSCR, these SNPs are the causal risk factors. Here as well, the remaining genes of the GRN are transcriptionally unchanged (Figure 4E) because RET expression never falls below $50 \%$.

\section{Discussion}

It is evident that a multiplicity of enhancers control a gene's expression: this feature has many implications for complex disease genetics and its mechanisms. The data reported here, based on human genetics, siRNA, and ChIP analyses in the SK-N-SH cell line, together with our prior studies ${ }^{2,11-13}$, have identified 38 distinct CREs of RET with sequence variants that are associated with HSCR. Of these, 10 contain common polymorphisms with differential allelic enhancer activity. CRISPR/Cas9 deletion analyses of these CREs demonstrate that at least 7 of these RET enhancers, binding the SOX10, RARB, GATA2, PAX3 and yet unknown TFs, have all of the hallmarks of harboring causal variants for HSCR (Table 3). Given that none of these experimental approaches are 100\% efficient yet other enhancers are likely. Nevertheless, the many enhancers at the RET locus, identified through in vitro analyses, do not imply that all of them are involved in gut (as opposed to other tissue) development, and, if so, whether they have unique roles in the ENS across development, as we have shown earlier (Chatterjee et al., 2016) or are merely shadow enhancers ${ }^{8}$. RET is highly expressed in the 
mid- and fore-brain, kidney and dorsal root ganglia: thus, these CREs may regulate RET in these tissues.

Definitive proof of our hypothesis that the 7 RET enhancers cause HSCR will require the creation of mouse models with multiple regulatory variants at the same locus, far more feasible using new methods of synthetic biology ${ }^{28}$ as opposed to CRISPR/Cas9 genome editing.

Nevertheless, this hypothesis is supported by the observation that RARB, GATA2, SOX10 and PAX3 binding to five of these enhancers have known roles in ENS development and HSCR 15,16,29. In other words, if the trans factors lead to HSCR and the cis factors that bind them are HSCR-associated then these cis factors are direct risk factors of HSCR. Their distinct nature and LD relationships also suggests that they contribute independently to RET gene expression, and, therefore to HSCR.

The multiplicity of noncoding variants in CREs all controlling the same gene should give us pause in interpreting the effect size and functional effects of individual GWAS variants. As we have shown, the cumulative effect of all variant-bearing enhancers is to significantly lower RET gene expression, as expected from RET coding mutations ${ }^{17,18}$. As we have also demonstrated, the largest risks are associated with haplotypes with multiple risk alleles, even across LD blocks ${ }^{2}$. This accumulation of multiple CRE variants is expected to lead to a larger effect through reduced binding of multiple TFs to multiple enhancers. Given the role of multiple TFs on the promoter this is likely synergistic. Additionally, other interactions are important in ENS cells with these genotypes. Note that RET also controls the gene expression of its own TFs PAX3, RARB, GATA2 and SOX10. This feedback may be a secondary but an important cause of reduced RET gene expression further exacerbating the enhancers' effect. We do not yet know whether this diversity of genetic control with feedback is typical or not. RET is a highly dosage-sensitive gene with higher and lower than wildtype levels being associated with neuroendocrine tumors and aganglionosis, respectively. Although extensive regulatory control is common for developmental genes ${ }^{30}$, the genetic lessons are likely to be universal.

The human genetic implications of these data, beyond understanding HSCR, are that genetically independent (unassociated) SNPs at a specific GWAS locus are not the only 
candidate variants for understanding a phenotype. Additional SNPs may be involved, even those perfectly associated with one another provided they affect functionally independent enhancers, as has been demonstrated by the recent discovery of additional RET variants and CREs that control its expression ${ }^{31,32}$. Finally, such regulatory control by the variant allele can also be quite varied (E4, E27 versus others), decreasing versus increasing enhancer activity, depending on activator versus repressor TFs and their co-regulators, as well as decreasing as well as increasing target gene expression. This suggests that understanding the regulatory contributions to GWAS will require experimental data on enhancer effects beyond what statistical analysis can suggest. Furthermore, we need broader enhancer screens to define the full enhancer architecture of RET and do so in vivo at different developmental stages and by sex. We also need to elucidate the full repertoire of TFs that regulate RET. These pieces of information are crucial to understand the full extent and composition of the RET-EDNRB GRN, which in turn will identify new genes that become mutational targets of HSCR. 


\section{Materials and methods}

Cell lines:

The human neuroblastoma cell SK-N-SH, purchased from ATCC, USA (\# HTB-11), was grown under standard conditions (DMEM $+10 \%$ FBS and 1\% Penicillin Streptomycin). It was maintained in $10 \mathrm{~cm}$ culture dishes and passaged every 48 hours when it reached $\sim 80 \%$ confluency.

\section{ChIP-seq peak calling:}

Three epigenomic datasets generated from a 108-day human fetal large intestine, histone H3K27ac ChIP-seq (GSM1058765), histone H3K4me1 ChIP-seq (GSM1058775), and DNasel-seq (GSM817188), were downloaded from the NIH Roadmap Epigenomics Project ${ }^{19}$. For the SK-N-SH cell line, DNasel-seq data (GSM736559) were obtained from the ENCODE project ${ }^{33}$. For each dataset, MACS software $\mathrm{v} 1.4{ }^{34}$ with default settings was used to call "peaks" at genomic sites where sequence reads were significantly enriched over background. With the default peak-calling threshold $\left(P<10^{-5}\right), 51,771,61,689,66,930$ and 52,534 genomic regions were identified in the GSM1058765, GSM1058775, GSM817188 and GSM736559 datasets, respectively.

Reporter assays:

$400 \mathrm{ng}$ of firefly luciferase vector ( $p$ GL4.23, Promega Corporation,USA) containing the DNA sequence of interest and $2 \mathrm{ng}$ of Renilla luciferase vector (transfection control) were transiently transfected into the SK-N-SH cell line $\left(5-6 \times 10^{4}\right.$ cells $/$ well $)$, using $6 \mathrm{ml}$ of FuGENE HD transfection reagent (Roche Diagnostic, USA) in $100 \mathrm{ml}$ of OPTIMEM medium (Invitrogen, USA). Cells were grown for 48 hours and luminescence measured using a Dual Luciferase Reporter Assay System on a Tecan multi-detection system luminometer, per the manufacturer's instructions.

Chromatin Immunoprecipitation-qPCR (ChIP-qPCR) assays:

ChIP was performed thrice independently for each antibody using $1 \times 10^{6} \mathrm{SK}-\mathrm{N}-\mathrm{SH}$ cells for each transcription factor using the EZ-Magna ChIP kit (Millipore Sigma, USA), as per the manufacturer's instructions, with the following modifications: the chromatin was sonicated for 
30s on and 30s off for 10 cycles; sheared chromatin was pre-blocked with unconjugated beads for 4 hours and specific antibodies separately conjugated to the beads for 4 hours before immunoprecipitation was performed with the pre-blocked chromatin. A polyclonal antibody was used against PAX3 (16HCLC; Invitrogen, USA) at 15 $\mathrm{g}$ concentration. ChIP assays were also performed on cells 48 hours after transfection with the PAX3 siRNAs (L-012399-00-0005; Dharmacon/Horizon Discovery, USA) at $25 \mu \mathrm{M}$ to assess specificity of TF binding. qPCR assays were performed using SYBR green (Life Technologies, USA) and specific primers against enhancer E2 (E2_FWD 5' GCTGCAGATATGCAACTTCCAA 3' and E2_REV 5' AGATATGCTGGTGAGGGGCT 3') and enhancer E27 (E27_FWD 5' AGGAAGGTAGGCACCCTGTA and E27_REV 5' AGCCCTGTGTTAACTGTCCG 3'). The data were normalized to input DNA and enrichment was calculated by fold excess over ChIP performed with specific IgG as background signal. All assays were done in triplicate for each independent ChIP assay $(n=9)$.

siRNA assays:

PAX3 (L-012399-00-0005), RET (L-003170-00-0005 ), SOX10 (L-017192-00), GATA2 (L-009024-02 and RARB (L-003438-02) SMARTpool siRNAs (combination of 4 distinct siRNAs targeting each gene) along with ON-TARGET plus non-targeting siRNAs (D001810-10, negative control) (Dharmacon/ Horizon Discovery, USA) were transfected at 20 $\mu \mathrm{M}$ in SK-N-SH cells at a density of $10^{4}-10^{5}$ cells using FuGene HD Transfection reagent (Promega Corporation, USA) per the manufacturer's instructions. Negative control siRNAs were always transfected at $25 \mu \mathrm{M}$ concentration.

Gene expression assays:

Total RNA was extracted from SK-N-SH cells using TRIzol (Life Technologies, USA) and cleaned on RNeasy columns (QIAGEN, USA). 500 $\mathrm{gg}$ of total RNA was converted to cDNA using SuperScriptIII reverse transcriptase (Life Technologies, USA) using Oligo-dT primers. The diluted (1/5) total cDNA was subjected to Taqman gene expression (ThermoFisher Scientific, USA) using the following transcript-specific probes and primers: $R E T$ (Hs01120032_m1), EDNRB (Hs00240747_m1), PAX3(Hs00240950_m1), SOX10 (Hs00366918_m1), GATA2 (Hs00231119_m1) and RARB (Hs00977140_m1) 
Human $\beta$-actin was used as an internal loading control for normalization. For siRNA knockdown experiments, five independent wells of SK-N-SH cells were used for RNA extraction and each assay performed in triplicate $(n=15)$. Relative fold change was calculated based on the $2 \Delta \Delta \mathrm{Ct}$ (threshold cycle) method. For siRNA experiments, $2 \Delta \Delta \mathrm{Ct}$ for negative control non-targeting control siRNA was set to unity. $P$ values were calculated from pairwise 2tailed t tests and the data presented as means with their standard errors (SE).

\section{CRIPSR/Cas9 induced deletions:}

\section{(i) Single guide disruptions}

Each enhancer region centered on a polymorphic site was targeted using a single guide RNA (Supplementary Table 2) by transfecting a ribonucleoprotein complex containing $100 \mathrm{pmol}$ of specific gRNA, coupled with $5 \mu \mathrm{g} / \mu \mathrm{l}$ of Truecut cas 9 nuclease (ThermoFisher Scientific) in Lipofectamine Crisprmax solution (ThermoFisher Scientific). For each enhancer, 5 wells containing $\sim 30,000$ SK-N-SH cells were independently transfected. To increase efficiency of deletion, we re-transfected the cells with the same ribonucleoprotein mix a second time after 72 hours. The cells were further grown for 48 hours and then equally split into 2 tubes for DNA and RNA extraction. To confirm disruption of the enhancer regions, specific primers (Supplementary Table 3) were used to verify deletions by PCR followed by Sanger sequencing. We used the Inference of CRISPR Edits (ICE) tool 24 to estimate the percentage of cells carrying various insertion/deletions.

\section{(ii) paired guide disruptions}

The following genomic regions were targeted with pairs of guides for 3 enhancers: RET-7 (RET-7_DGR-5'GACCCCTCTGGACCCGTCGCGG3 and RET-2_UGRCCGAGTGTTTGCGCCCATGAGG), RET-5.5 (RET-5.5_DGR-

AAGGATGGATACACCTTCCGGG and RET-5.5_UGR-AAGGATGGATACACCTTCCGGG) and RET+3 (RET+3_DGR-GCCTGGCTGGAGGTCCAAGAGG and RET+3_UGRGCTCATGAGGAGCACACCGTGG). The guide RNA and Cas9 were cloned into the vector U6-gRNA/CMV-Cas9-GFP (Millipore Sigma, USA) and each pair of guides were transfected at $10 \mu \mathrm{g}$ into 72 wells of low-density ( 10,000 cells) plated SK-N-SH cells. We also transfected 72 wells with only the Cas9-GFP vector without the guide RNAs to serve as 
controls against which to measure gene expression changes arising from our enhancer deletions.

After 24 hours cells were checked for GFP expression and wells with at least $\sim 80 \%$ positive cells were re-plated, ensuring high transfection efficiency. For all 3 constructs and the control we obtained at least 48 wells with over $80 \%$ transfection efficiency. The cells were further grown for 24 hours and then split into 2 tubes of equal cells for DNA and RNA extractions.

To check for deletions, we used the following pairs of primers: RET-7 (RET-7_FWD 5' GAGGAACCGCGCACATCAG 3' and RET-7_REV 5' CGCCTTGCCTGGCCCTCCA 3'), RET-5.5 (RET-5.5_FWD 5' GTCCTGGTTGCCCCACTCTG 3' and RET-5.5_REV 5' GGTCAGGTGTCACAAAGTCT 3') and RET+3 (RET+3_FWD 5'

AATGGGCAAGACCATCTCAG 3' and RET+3_REV 5' GTGGCCAGTAGCTGGAAGAGCAG 3'). The primers amplify a wildtype sequence of $1.1 \mathrm{~kb}$ for each enhancer region. Each cell line was PCR confirmed for enhancer deletion and Sanger sequenced to re-confirm the precise region of deletion. For quantifying gene expression changes, RNA was extracted from each cell line with deletions and qPCR assays performed in triplicate; $n=6$ for RET-7 and $n=9$ for RET-5.5 and RET+3. Quantitative gene expression changes were calculated as detailed above.

\section{Estimating haplotype-specific HSCR risk:}

Genotypes at 10 RET CRE variants in 220 S-HSCR cases and 503 Europeanancestry controls were obtained from our published HSCR Genome Wide Association study ${ }^{14}$ and the 1000 Genomes project ${ }^{21}$, respectively. Haplotypes were generated from unphased genotypes using BEAGLE ${ }^{35}$ and filtered to retain only those that had a frequency $>1 \%$ in controls. Standard methods using $\chi^{2}$ statistics were used to calculate haplotype-count based odds ratios (OR), their upper and lower confidence limits and significance of their deviation from the null hypothesis of no association $(\mathrm{OR}=1)^{13}$. 
Identifying transcription factors for candidate enhancers:

We searched for TF binding sites (TFBS) within all putative CREs using FIMO ${ }^{36,37}$ and 890 validated TF motifs in TRANSFAC ${ }^{38}$. We used the setting of "minimize false positives" and a stringent cut off of $\mathrm{P}<10^{-4}$ to identify candidate cognate TFs.

\section{Author Contributions:}

S.C and A.C. conceived and designed the study. K.M.K. conducted all in vitro luciferase assays, A.K. all genotyping assays and S.C and L.E.F all in vivo CRISPR assays. S.C. and A.C. wrote and edited the manuscript.

\section{Funding:}

This work was supported by a National Institute of Health MERIT Award HD28088 to AC. 


\section{REFERENCES}

1. Visscher, P.M. et al. 10 Years of GWAS Discovery: Biology, Function, and Translation. Am J Hum Genet 101, 5-22 (2017).

2. Chatterjee, S. et al. Enhancer Variants Synergistically Drive Dysfunction of a Gene Regulatory Network In Hirschsprung Disease. Cell 167, 355-368 e10 (2016).

3. Chakravarti, A. \& Turner, T.N. Revealing rate-limiting steps in complex disease biology: The crucial importance of studying rare, extreme-phenotype families. Bioessays 38, 578-86 (2016).

4. Maurano, M.T. et al. Systematic localization of common disease-associated variation in regulatory DNA. Science 337, 1190-5 (2012).

5. Dixon, J.R. et al. Topological domains in mammalian genomes identified by analysis of chromatin interactions. Nature 485, 376-80 (2012).

6. Rao, S.S. et al. A 3D map of the human genome at kilobase resolution reveals principles of chromatin looping. Cell 159, 1665-80 (2014).

7. Chatterjee, S. \& Ahituv, N. Gene Regulatory Elements, Major Drivers of Human Disease. Annu Rev Genomics Hum Genet 18, 45-63 (2017).

8. Kvon, E.Z., Waymack, R., Elabd, M.G. \& Wunderlich, Z. Enhancer redundancy in development and disease. Nat Rev Genet (2021).

9. Kapoor, A. et al. Multiple SCN5A variant enhancers modulate its cardiac gene expression and the QT interval. Proc Natl Acad Sci U S A 116, 10636-10645 (2019).

10. Inoue, F., Kreimer, A., Ashuach, T., Ahituv, N. \& Yosef, N. Identification and Massively Parallel Characterization of Regulatory Elements Driving Neural Induction. Cell Stem Cell 25, 713-727 e10 (2019).

11. Tilghman, J.M. et al. Molecular Genetic Anatomy and Risk Profile of Hirschsprung's Disease. N Engl J Med 380, 1421-1432 (2019).

12. Chatterjee, S. \& Chakravarti, A. A gene regulatory network explains RET-EDNRB epistasis in Hirschsprung disease. Hum Mol Genet 28, 3137-3147 (2019).

13. Kapoor, A. et al. Population variation in total genetic risk of Hirschsprung disease from common RET, SEMA3 and NRG1 susceptibility polymorphisms. Hum Mol Genet 24, 2997-3003 (2015). 
14. Jiang, Q. et al. Functional loss of semaphorin 3C and/or semaphorin 3D and their epistatic interaction with ret are critical to Hirschsprung disease liability. Am J Hum Genet 96, 581-96 (2015).

15. Bondurand, N. et al. Interaction among SOX10, PAX3 and MITF, three genes altered in Waardenburg syndrome. Hum Mol Genet 9, 1907-17 (2000).

16. Lang, D. et al. Pax3 is required for enteric ganglia formation and functions with Sox10 to modulate expression of c-ret. J Clin Invest 106, 963-71 (2000).

17. Emison, E.S. et al. A common sex-dependent mutation in a RET enhancer underlies Hirschsprung disease risk. Nature 434, 857-63 (2005).

18. Emison, E.S. et al. Differential contributions of rare and common, coding and noncoding Ret mutations to multifactorial Hirschsprung disease liability. Am J Hum Genet 87, 60-74 (2010).

19. Bernstein, B.E. et al. The NIH Roadmap Epigenomics Mapping Consortium. Nat Biotechnol 28, 1045-8 (2010).

20. Consortium, E.P. An integrated encyclopedia of DNA elements in the human genome. Nature 489, 57-74 (2012).

21. Genomes Project, C. et al. A global reference for human genetic variation. Nature 526, 68-74 (2015).

22. Chatterjee, S., Nandakumar, P., Auer, D.R., Gabriel, S.B. \& Chakravarti, A. Gene- and tissue-level interactions in normal gastrointestinal development and Hirschsprung disease. Proc Natl Acad Sci U S A (2019).

23. Natarajan, D., Marcos-Gutierrez, C., Pachnis, V. \& de Graaff, E. Requirement of signalling by receptor tyrosine kinase RET for the directed migration of enteric nervous system progenitor cells during mammalian embryogenesis.

Development 129, 5151-60 (2002).

24. Hsiau, T. et al. Inference of CRISPR Edits from Sanger Trace Data. bioRxiv, 251082 (2019).

25. Pasini, B. et al. Loss of function effect of RET mutations causing Hirschsprung disease. Nat Genet 10, 35-40 (1995).

26. Angrist, M. et al. Mutation analysis of the RET receptor tyrosine kinase in Hirschsprung disease. Hum Mol Genet 4, 821-30 (1995).

27. Uesaka, T., Nagashimada, M., Yonemura, S. \& Enomoto, H. Diminished Ret expression compromises neuronal survival in the colon and causes intestinal aganglionosis in mice. J Clin Invest 118, 1890-8 (2008). 
28. Richardson, S.M. et al. Design of a synthetic yeast genome. Science 355, 10401044 (2017).

29. Lang, D. \& Epstein, J.A. Sox10 and Pax3 physically interact to mediate activation of a conserved c-RET enhancer. Hum Mol Genet 12, 937-45 (2003).

30. Bolt, C.C. \& Duboule, D. The regulatory landscapes of developmental genes. Development 147(2020).

31. Fu, A.X. et al. Whole-genome analysis of noncoding genetic variations identifies multiscale regulatory element perturbations associated with Hirschsprung disease. Genome Res 30, 1618-1632 (2020).

32. Kapoor, A. et al. Multiple, independent, common variants overlapping known and putative gut enhancers at $<$ em $>$ RET $</$ em $>$, $<$ em $>$ SEMA3 $</$ em $>$ and $<\mathrm{em}>\mathrm{NRG} 1</ \mathrm{em}>$ underlie Hirschsprung disease risk in European ancestry subjects. bioRxiv, 2020.06.07.138719 (2020).

33. Consortium, E.P. et al. Expanded encyclopaedias of DNA elements in the human and mouse genomes. Nature 583, 699-710 (2020).

34. Zhang, Y. et al. Model-based analysis of ChIP-Seq (MACS). Genome Biol 9, R137 (2008).

35. Browning, S.R. \& Browning, B.L. Rapid and accurate haplotype phasing and missing-data inference for whole-genome association studies by use of localized haplotype clustering. Am J Hum Genet 81, 1084-97 (2007).

36. Bailey, T.L. et al. MEME SUITE: tools for motif discovery and searching. Nucleic Acids Res 37, W202-8 (2009).

37. Grant, C.E., Bailey, T.L. \& Noble, W.S. FIMO: scanning for occurrences of a given motif. Bioinformatics 27, 1017-8 (2011).

38. Wingender, E., Dietze, P., Karas, H. \& Knuppel, R. TRANSFAC: a database on transcription factors and their DNA binding sites. Nucleic Acids Res 24, 238-41 (1996). 


\section{Figure Legends}

Figure 1: The RET regulatory landscape in the enteric nervous system. (A) The $155 \mathrm{~kb}$ RET locus (10:43434933-43590368; hg19) contains 38 putative cis regulatory elements (CRE) with HSCR-associated polymorphisms in 4 linkage disequilibrium (LD) blocks. Multiple enhancer-associated epigenetic marks (DHS: DNasel hypersensitivity, H3K27ac, $\mathrm{H} 3 \mathrm{~K} 4 \mathrm{me}$ 1) in 108-day human fetal large intestine and the SK-N-SH neuroblastoma cell line, and transcription factor (TF) binding sites, from public sources, are noted. All common ( $\geq 10 \%$ allele frequency) variants associated with HSCR are shown, with those showing allelic difference in in vitro transcription assays marked in red (this paper). (B) Allele specific in vitro luciferase assays of the 38 CREs in (A) in SK-N-SH cells are shown: 22 CREs act as enhancers, as compared to a promoter-only control, of which, 7 also show allelic difference in luciferase activity (boxed) for its cognate HSCR-associated polymorphism in addition to RET-7, RET-5.5 and RET+3 positive controls. Error bars are standard errors (SE) of the mean $\left({ }^{*} \mathrm{P}<0.01,{ }^{* *} \mathrm{P}<0.001\right)$ for 3 independent biological replicates for each assay.

Figure 2: Identification of cognate transcription factors bound to RET enhancers. Genome map of the RET locus with locations of the E2 and E27 CREs together with ChIPqPCR results using a PAX3 antibody in SK-N-SH cells shows enrichment of binding as compared to the background. The specificity of binding is shown by siRNA knockdown of $P A X 3$ with concomitant reduction in ChIP-qPCR signals at both CREs. Error bars are standard errors (SE) of the mean $\left({ }^{*} \mathrm{P}<0.01,{ }^{* *} \mathrm{P}<0.001\right)$ for 3 independent biological replicates for each ChIP assay.

Figure 3: Transcription factor-mediated in vitro and in vivo effects on gene expression.

(A) siRNA-mediated knockdown of PAX3, SOX10, GATA2, RARB and RET in SK-N-SH cells decreases RET gene expression. (B) siRNA-mediated knockdown of PAX3 has significant transcriptional effects on SOX10 but small yet statistically insignificant effects on 
GATA2 and RARB. Error bars are standard errors (SE) of the mean $\left({ }^{*} \mathrm{P}<0.01,{ }^{*} \mathrm{P}<\right.$ 0.001 ) for 5 independent biological replicates in all experiments.

Figure 4: CRISPR/Cas9 induced deletions of RET enhancers with HSCR-associated variants reduce RET gene expression in vitro. (A) There is significant loss of RET gene expression from 7 of 9 CREs with small $(\leq 10 \mathrm{bp})$ deletions centered on the variant site; only the E14 enhancer shows increased gene expression. (B) There is significant loss of average RET expression from the cumulative effect of individual enhancer deletions as compared to wildtype cells. (C) The expression of other RET GRN genes are unaffected by these CRE deletions likely owing to RET expression never decreasing below $50 \%$. (D) A genomic map of the RET locus showing 3 positive control CREs and their corresponding HSCR-associated polymorphisms: RET-7 (rs2506030), RET-5.5 (rs7069590) and RET+3 (rs2435357). The green boxed region is the deleted region for each enhancer as confirmed by Sanger sequencing and validated by agarose gel electrophoresis. (E) Heterozygous deletion of all 3 enhancers lead to significant loss of RET gene expression but not other $R E T$ GRN genes. Error bars are standard errors (SE) of the mean ( ${ }^{*} P<0.01$, ${ }^{*} P<0.001$ ) for 5 independent biological replicates in all experiments for single guide deletions, across 6 replicates for RET-7 and 9 replicates for RET-5.5 and RET +3 in dual guide deletions. 


\section{$\underline{\text { TABLES }}$}

\begin{tabular}{|c|c|c|c|c|c|c|}
\hline \multirow{2}{*}{$\begin{array}{l}\text { Genomic } \\
\text { element }\end{array}$} & \multirow{2}{*}{ SNP ID } & \multicolumn{2}{|c|}{ Genomic location (chr10) } & \multirow[t]{2}{*}{ LD Block } & \multirow{2}{*}{$\begin{array}{l}\text { Non-Risk } \\
\text { Allele }\end{array}$} & \multirow{2}{*}{$\begin{array}{l}\text { Risk } \\
\text { Allele }\end{array}$} \\
\hline & & Start & End & & & \\
\hline E1 & rs788267 & 43434932 & 43435936 & \multirow{12}{*}{ Block 1} & $\mathrm{C}$ & $T$ \\
\hline E2 & rs788263 & 43437007 & 43437506 & & C & G \\
\hline E3 & rs788262 & 43437440 & 43437943 & & $A$ & G \\
\hline E4 & rs788261 & 43437726 & 43438225 & & $T$ & C \\
\hline E5 & rs788260 & 43438228 & 43438727 & & G & A \\
\hline E6 & rs2995411 & 43440554 & 43441053 & & $\mathrm{C}$ & $T$ \\
\hline E7 & rs1582227 & 43441446 & 43441952 & & $T$ & C \\
\hline E8 & rs2488278 & 43446082 & 43446581 & & $T$ & $\mathrm{C}$ \\
\hline RET-7 & rs2506030 & 43447620 & 43448074 & & A & G \\
\hline E9 & rs $17158318 /$ rs 17158320 & 43448581 & 43449090 & & $\mathrm{G} / \mathrm{C}$ & $\mathrm{A} / \mathrm{A}$ \\
\hline E10 & rs947696 & 43455083 & 43455582 & & G & $\mathrm{T}$ \\
\hline E11 & rs7908085 & 43460567 & 43461065 & & $T$ & A \\
\hline E12 & rs10900290 & 43471322 & 43471823 & \multirow{4}{*}{ Block 2} & C & $T$ \\
\hline E13 & rs947690 & 43479479 & 43479979 & & G & C \\
\hline E14 & rs1547930 & 43483056 & 43483559 & & G & A \\
\hline E15 & rs3004258 & 43483900 & 43484403 & & $T$ & G \\
\hline RET-5.5 & rs7069590 & 43552669 & 43553121 & \multirow{4}{*}{ Block 3} & $C$ & $T$ \\
\hline E16 & rs3026703 & 43557546 & 43558048 & & $T$ & $C$ \\
\hline E17 & rs3026707 & 43558368 & 43559122 & & A & G \\
\hline E18 & rs2505989 & 43563195 & 43563700 & & G & C \\
\hline RET+3 & rs2435357 & 43581829 & 43582283 & \multirow{5}{*}{ Block 4} & C & $T$ \\
\hline E19 & rs741763 & 43568087 & 43568586 & & G & C \\
\hline E20 & rs2505995 & 43569379 & 43569878 & & A & G \\
\hline E21 & rs2506010 & 43573167 & 43574025 & & $C$ & $T$ \\
\hline E22 & rs2506020 & 43578754 & 43579373 & & $C$ & $T$ \\
\hline E23 & rs2506021/ rs2435342 & 43583869 & 43584408 & Block 5 & $\mathrm{C} / \mathrm{T}$ & $T / C$ \\
\hline E24 & rs2506022 & 43584264 & 43584845 & \multirow{5}{*}{ Block 6} & $\mathrm{C}$ & $\mathrm{T}$ \\
\hline E25 & rs2435343 & 43585384 & 43585889 & & $T$ & G \\
\hline E26 & rs12247456 & 43587982 & 43588420 & & G & A \\
\hline E27 & rs7393733 & 43588440 & 43588915 & & G & C \\
\hline E28 & rs2505541 & 43589862 & 43590368 & & $T$ & $C$ \\
\hline
\end{tabular}

Table 1: Genomic coordinates (hg19) of 31 elements containing 33 single nucleotide polymorphisms (SNPs), within 6 linkage disequilibrium (LD) blocks at the RET locus which are associated with Hirschsprung disease (HSCR). 25 elements in bold act as enhancers in in vitro transcriptional assays while 10 SNPs in bold show allelic difference between the risk and non-risk allele as well. Enhancer activities of elements RET-7 (rs2506030), RET-5.5 (rs7069590) and RET+3 (rs2435357) have been previously demonstrated to be affected by the HSCR associated risk alleles ${ }^{2}$. 


\begin{tabular}{|c|c|c|c|c|}
\hline Haplotype & $\begin{array}{l}\text { Case } \\
\text { Frequency }\end{array}$ & $\begin{array}{l}\text { Control } \\
\text { Frequency }\end{array}$ & $\begin{array}{l}\text { Odds Ratio (95\% } \\
\mathrm{Cl})\end{array}$ & $\mathbf{P}$ \\
\hline CTGAACCACT & 0.02 & 0.08 & 1 & 1 \\
\hline CTGAATCACT & 0.04 & 0.07 & $2.24(0.95-5.29)$ & 0.07 \\
\hline CTGAGCCACT & 0.03 & 0.10 & $1.13(0.45-2.82)$ & 0.79 \\
\hline CTGAATCGGC & 0.04 & 0.06 & $2.84(1.20-6.77)$ & 0.02 \\
\hline CTGAGTCGGC & 0.06 & 0.16 & $1.58(0.71-3.51)$ & 0.26 \\
\hline CTGAATTGGT & 0.02 & 0.01 & $4.35(1.41-13.49)$ & 0.01 \\
\hline CTGAGTTGGT & 0.10 & 0.06 & $7.2(3.26-15.91)$ & $1.02 \times 10^{-6}$ \\
\hline GCAGGTCACT & 0.02 & 0.04 & $2.45(0.92-6.54)$ & 0.07 \\
\hline GCAGGTCGGC & 0.06 & 0.15 & $1.62(0.72-3.61)$ & 0.24 \\
\hline GCAGGTTGGT & 0.52 & 0.17 & $12.20(5.97-24.93)$ & $7.02 \times 10^{-12}$ \\
\hline
\end{tabular}

Table 2: Haplotypes for ten RET enhancer polymorphisms (rs788263, rs788261, rs788260, rs2506030, rs1547930, rs7069590, rs2435357, rs12247456, rs7393733 and rs2505541), with risk alleles denoted in bold. Observed frequencies of haplotypes among 220 European ancestry HSCR cases and 503 controls together with the odds ratio (with respect to the reference haplotype CTGAACCACT containing only one susceptibility allele; significant values bolded) and statistical significance $(P)$ are shown. 


\begin{tabular}{|c|c|c|c|c|c|c|c|c|c|c|}
\hline \multirow{3}{*}{$\begin{array}{l}\# \\
1 \\
\end{array}$} & \multirow{3}{*}{$\begin{array}{r}\text { SNP ID } \\
\text { rs788267 }\end{array}$} & \multirow[t]{2}{*}{$\begin{array}{l}\text { LD } \\
\text { Block }\end{array}$} & \multirow{2}{*}{$\begin{array}{c}\text { DNA } \\
\text { element }\end{array}$} & \multicolumn{2}{|c|}{$\begin{array}{l}\text { Coordinates } \\
\text { (chr 10, hg19) }\end{array}$} & \multirow{3}{*}{$\begin{array}{c}\begin{array}{c}\text { Epigenetic } \\
\text { marks }\end{array} \\
+^{a}\end{array}$} & \multirow{3}{*}{$\begin{array}{c}\text { Reporter } \\
\text { assay }\end{array}$} & \multirow{3}{*}{$\begin{array}{c}\begin{array}{c}\text { Risk } \\
\text { allele } \\
\text { effect }\end{array} \\
-\end{array}$} & \multirow{3}{*}{$\begin{array}{l}\text { TF } \\
-\end{array}$} & \multirow{3}{*}{$\begin{array}{c}\begin{array}{c}R E T \\
\text { expression }\end{array} \\
\text { NT }\end{array}$} \\
\hline & & & & Start & Stop & & & & & \\
\hline & & \multirow{13}{*}{$\begin{array}{c}\text { Block } \\
1\end{array}$} & E1 & 43434932 & 43435936 & & & & & \\
\hline 2 & rs788263 & & E2 & 43437007 & 43437506 & $+^{a}$ & + & increase & PAX3 & no change \\
\hline 3 & rs788262 & & E3 & 43437440 & 43437943 & $+^{a}$ & + & - & - & NT \\
\hline 4 & rs788261 & & E4 & 43437726 & 43438225 & $+^{a}$ & + & increase & - & increase \\
\hline 5 & rs788260 & & E5 & 43438228 & 43438727 & - & + & + & - & no change \\
\hline 6 & rs2995411 & & E6 & 43440554 & 43441053 & - & + & - & - & NT \\
\hline 7 & rs1582227 & & E7 & 43441446 & 43441952 & - & + & - & - & NT \\
\hline 8 & rs2488278 & & E8 & 43446082 & 43446581 & - & + & - & - & NT \\
\hline $9^{*}$ & rs2506030 & & RET-7 & 43447346 & 43448347 & $+^{a},+^{b}$ & + & decrease & RARB & decreased \\
\hline 10 & rs 17158318 & & E9 & 43448581 & 43449090 & $+^{a},+^{b}$ & + & - & - & NT \\
\hline 11 & rs 17158320 & & E9 & \multicolumn{2}{|c|}{ Included with SNP 10} & $+a,+b$ & + & - & - & NT \\
\hline 12 & rs947696 & & E10 & 43455083 & 43455582 & - & - & - & - & NT \\
\hline 13 & rs7908085 & & E11 & 43460567 & 43461065 & - & + & - & - & NT \\
\hline 14 & rs10900290 & & $\mathrm{E} 12$ & 43471322 & 43471823 & - & + & - & - & NT \\
\hline 15 & rs947690 & \multirow{3}{*}{$\begin{array}{l}\text { Block } \\
2\end{array}$} & E13 & 43479479 & 43479979 & - & - & - & - & NT \\
\hline 16 & rs1547930 & & E14 & 43483056 & 43483559 & $++^{a}$ & + & decrease & - & no change \\
\hline 17 & rs3004258 & & E15 & 43483900 & 43484403 & - & + & - & - & NT \\
\hline 18 & rs4948702 & \multirow{5}{*}{$\begin{array}{c}\text { Block } \\
3\end{array}$} & rs4948702 & 43551663 & 43552663 & $+^{a}$ & + & - & - & NT \\
\hline $19^{*}$ & rs7069590 & & RET-5.5 & 43552669 & 43553121 & $+^{a}$ & + & decrease & GATA2 & decreased \\
\hline 20 & rs3026703 & & E16 & 43557546 & 43558048 & - & + & - & - & NT \\
\hline 21 & rs3026707 & & E17 & 43558368 & 43559122 & - & + & - & - & NT \\
\hline 22 & rs2505989 & & E18 & 43563195 & 43563700 & - & + & - & - & NT \\
\hline 23 & rs2435367 & & rs2435367 & 43566114 & 43567114 & - & + & - & - & NT \\
\hline 24 & rs741763 & \multirow{6}{*}{$\begin{array}{c}\text { Block } \\
4\end{array}$} & E19 & 43568087 & 43568586 & - & + & - & - & NT \\
\hline 25 & rs2505995 & & E20 & 43569379 & 43569878 & - & + & - & - & NT \\
\hline 26 & rs2506010 & & E21 & 43573167 & 43574025 & $+^{a}$ & + & - & - & NT \\
\hline 27 & rs2506011 & & rs2506011 & 43574436 & 43575436 & $+^{a}$ & + & - & - & NT \\
\hline 28 & rs2506020 & & E22 & 43578754 & 43579373 & - & - & - & - & NT \\
\hline $29^{*}$ & rs2435357 & & RET+3 & 43581829 & 43582283 & $+^{a},+^{b}$ & + & decrease & SOX10 & decreased \\
\hline 30 & rs2506021 & \multirow{2}{*}{$\begin{array}{c}\text { Block } \\
5\end{array}$} & E23 & 43583869 & 43584408 & - & - & - & - & NT \\
\hline 31 & rs2435342 & & E23 & \multicolumn{2}{|c|}{ Included with SNP 30} & - & - & - & - & NT \\
\hline 32 & rs2506022 & \multirow{7}{*}{$\begin{array}{c}\text { Block } \\
6\end{array}$} & E24 & 43584264 & 43584845 & - & - & - & - & NT \\
\hline 33 & rs2435343 & & E25 & 43585384 & 43585889 & - & + & - & - & NT \\
\hline 34 & rs12247456 & & E26 & 43587982 & 43588420 & $+^{a}$ & + & decrease & - & decreased \\
\hline 35 & rs752978 & & rs752978 & 43587929 & 43588929 & - & + & - & - & NT \\
\hline 36 & rs7393733 & & E27 & 43588440 & 43588915 & $+^{a}$ & + & increase & PAX3 & decreased \\
\hline 37 & rs2506024 & & rs2506024 & 43588418 & 43589418 & - & + & - & - & NT \\
\hline 38 & rs2505541 & & E28 & 43589862 & 43590368 & $+^{a}$ & + & increase & - & decreased \\
\hline
\end{tabular}

Table 3: 38 Hirschsprung disease (HSCR)-associated polymorphisms in 6 linkage disequilibrium (LD) blocks, contained within 36 DNA elements at the RET locus annotated with respect to epigenetic marks (DNasel hypersensitivity $\left(+^{a}\right)$ in the SK-N-SH cell line, H3K4me1 $\left(+^{b}\right)$ marks in human fetal gut), luciferase reporter assays of alleles in the SK-N-SH cell line, allelic differences 
in luciferase assays in the SK-N-SH cell line, the transcription factor (TF) binding the indicated regulatory element, and whether deletion of the element affected RET gene expression. 31 elements have enhancer activity in in vitro luciferase assays, 10 of which demonstrated affect due to the HSCR associated risk polymorphisms. CRISPR based in vivo deletion of these 10 enhancers identified 7 which affect RET gene expression, 5 of which are bound by PAX3, RARB, GATA2 and SOX10 TFs. Elements \#9, 18, 19, 23, 27, 29, 35 and 37 were reported in our previous study ${ }^{2}$ and are included for completeness; of these 9,19 and 29, marked by *, are positive controls with in vivo evidence of enhancer activity in transgenic mice. NT: not tested. 
bioRxiv preprint doi: https://doi.org/10.1101/2020.06.18.159459; this version posted April 10, 2021. The copyright holder for this preprint (which
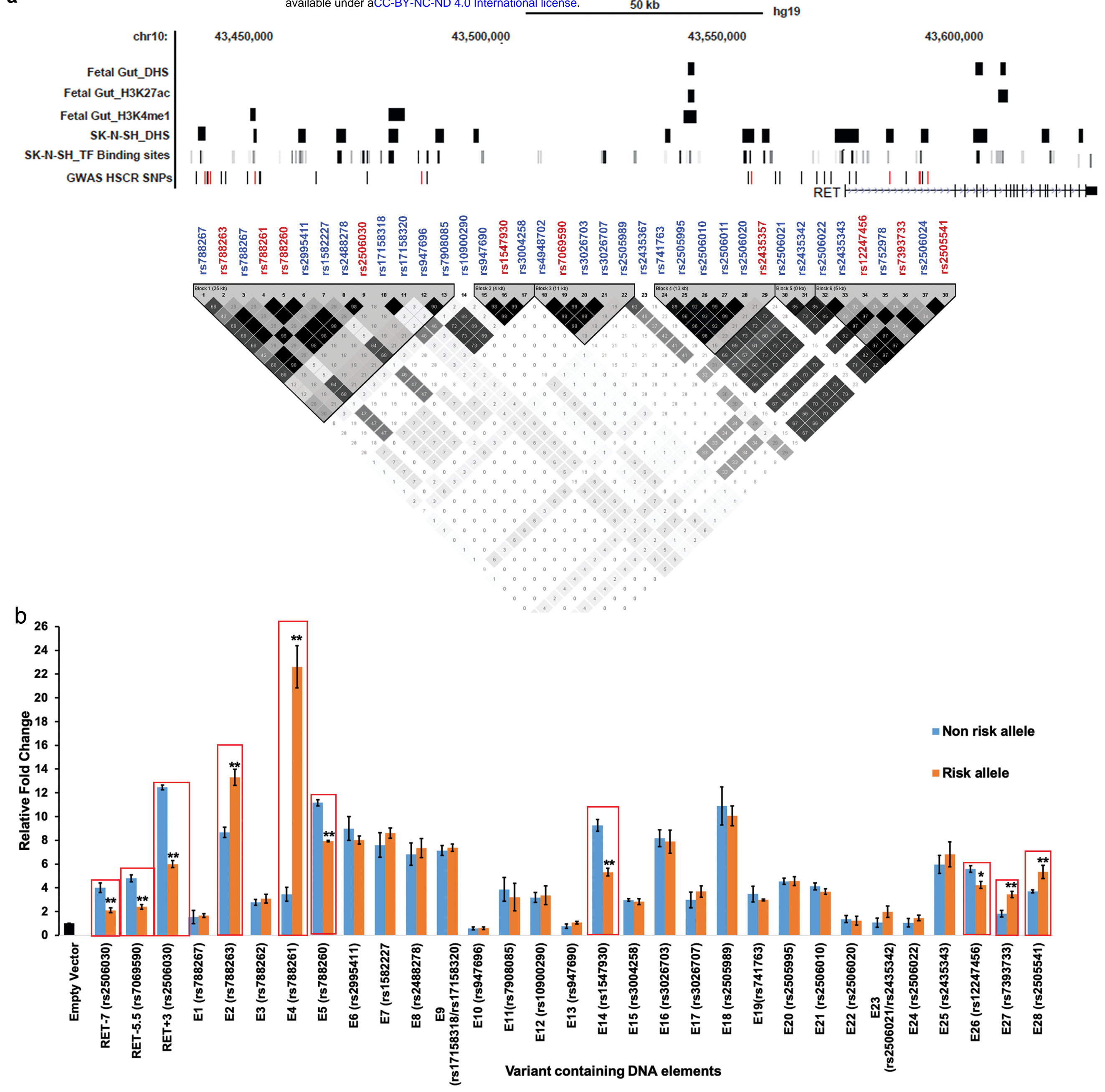


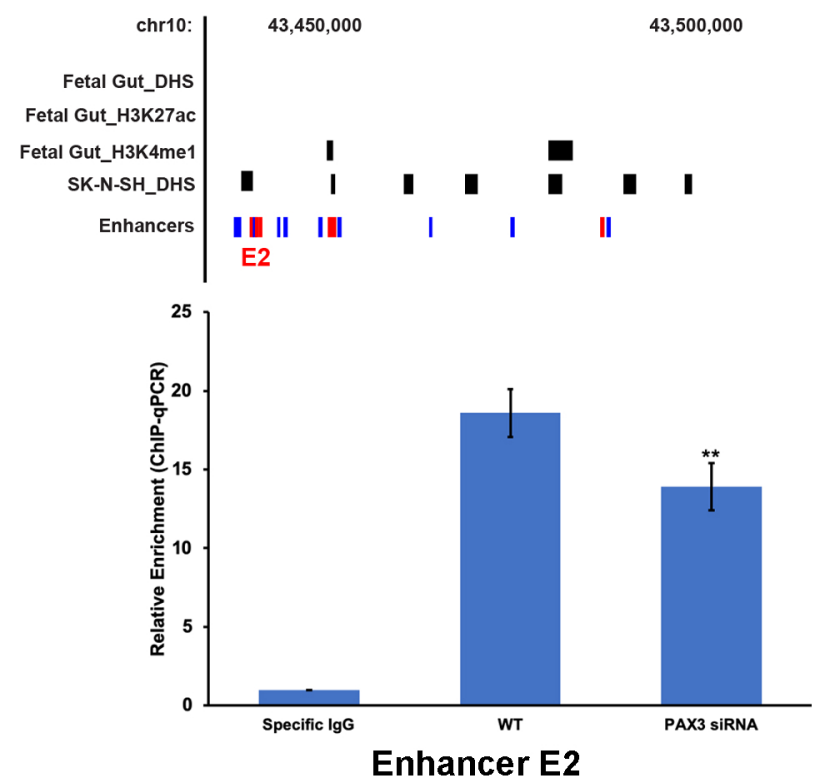

$43,550,000$

$43,600,000$
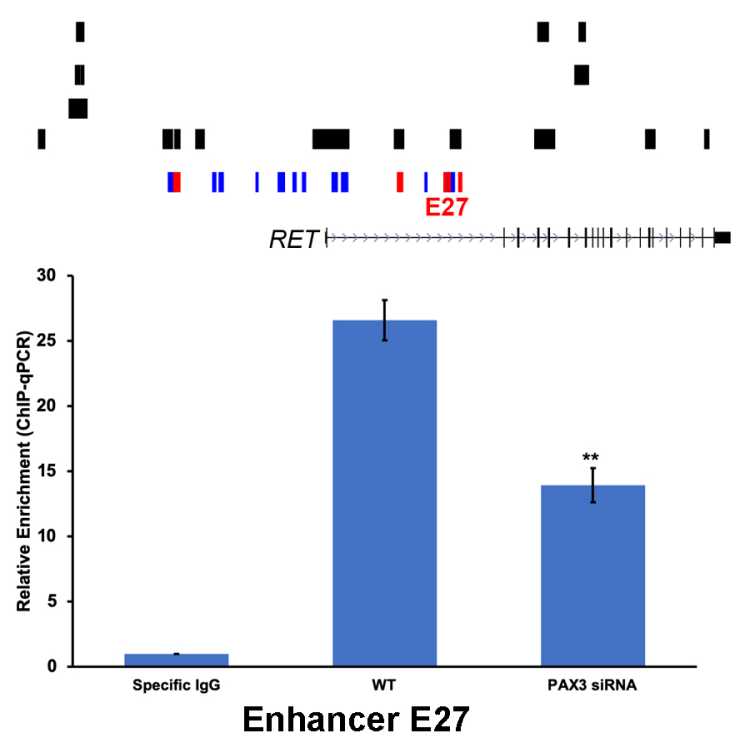


\section{bioRxiv preprint doi: https://doi.org/10.1101/2020.06.18.159459; this version posted April 10, 2021. The copyright holder for this preprint (which}
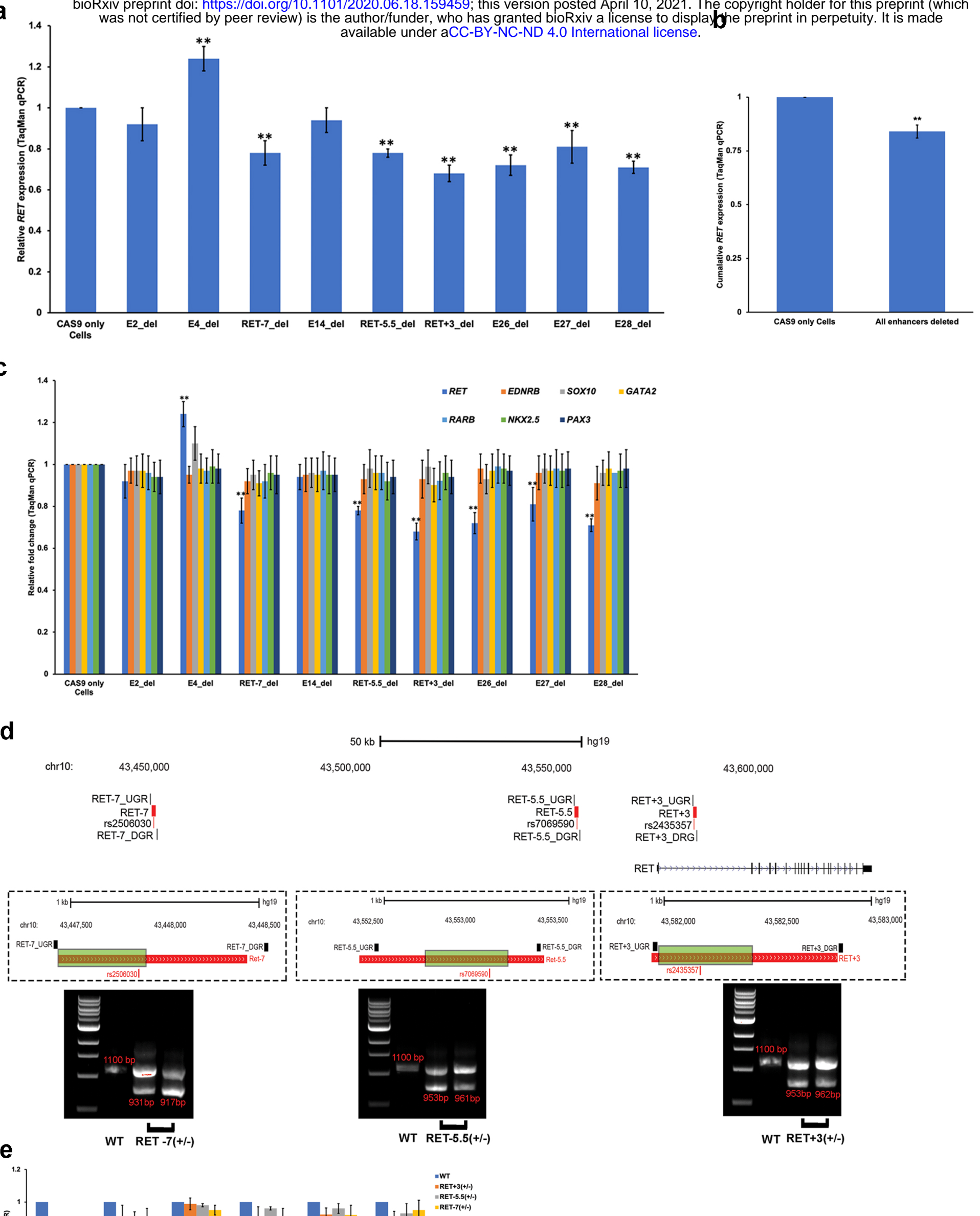

C

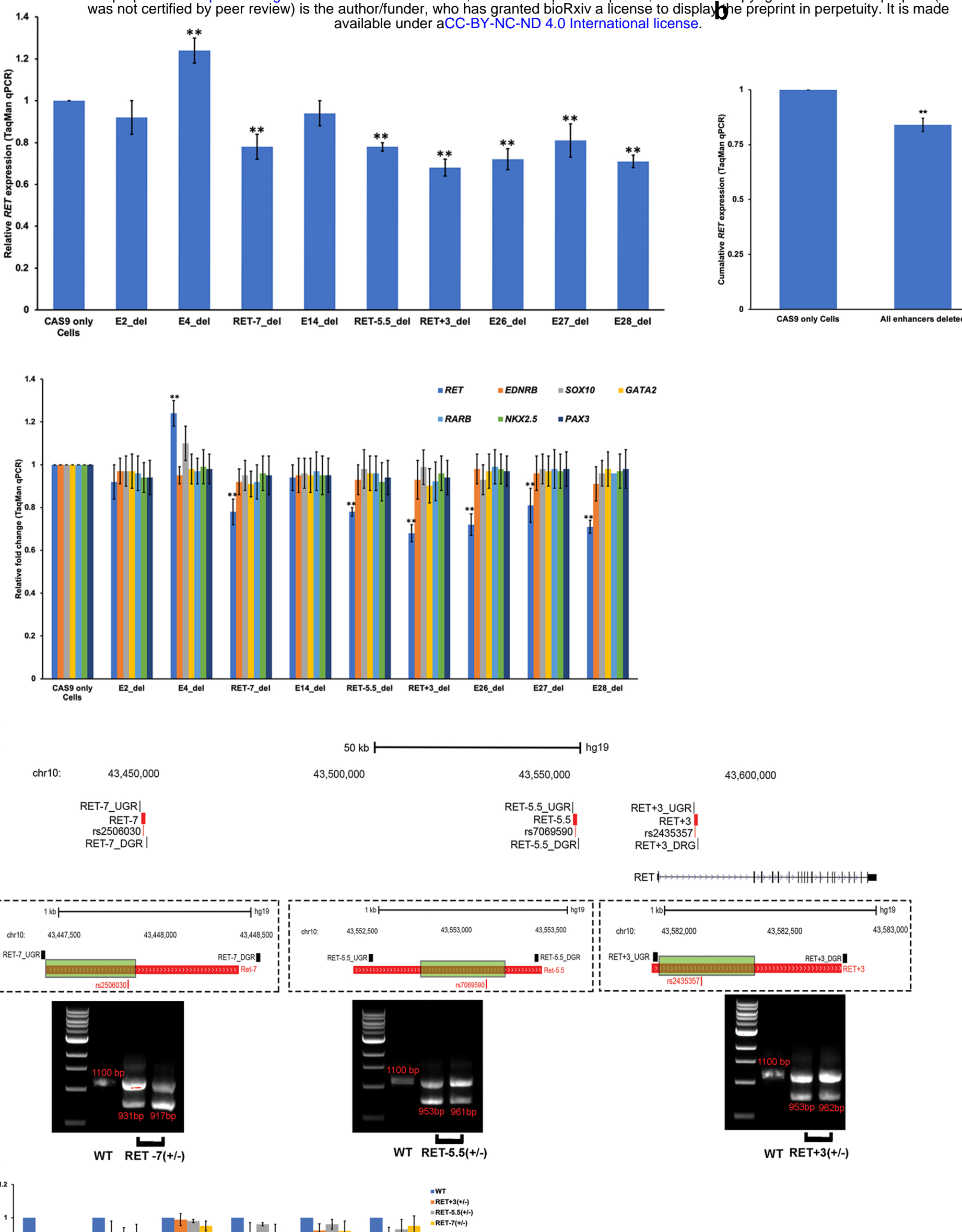

d

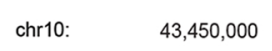

$50 \mathrm{~kb}$

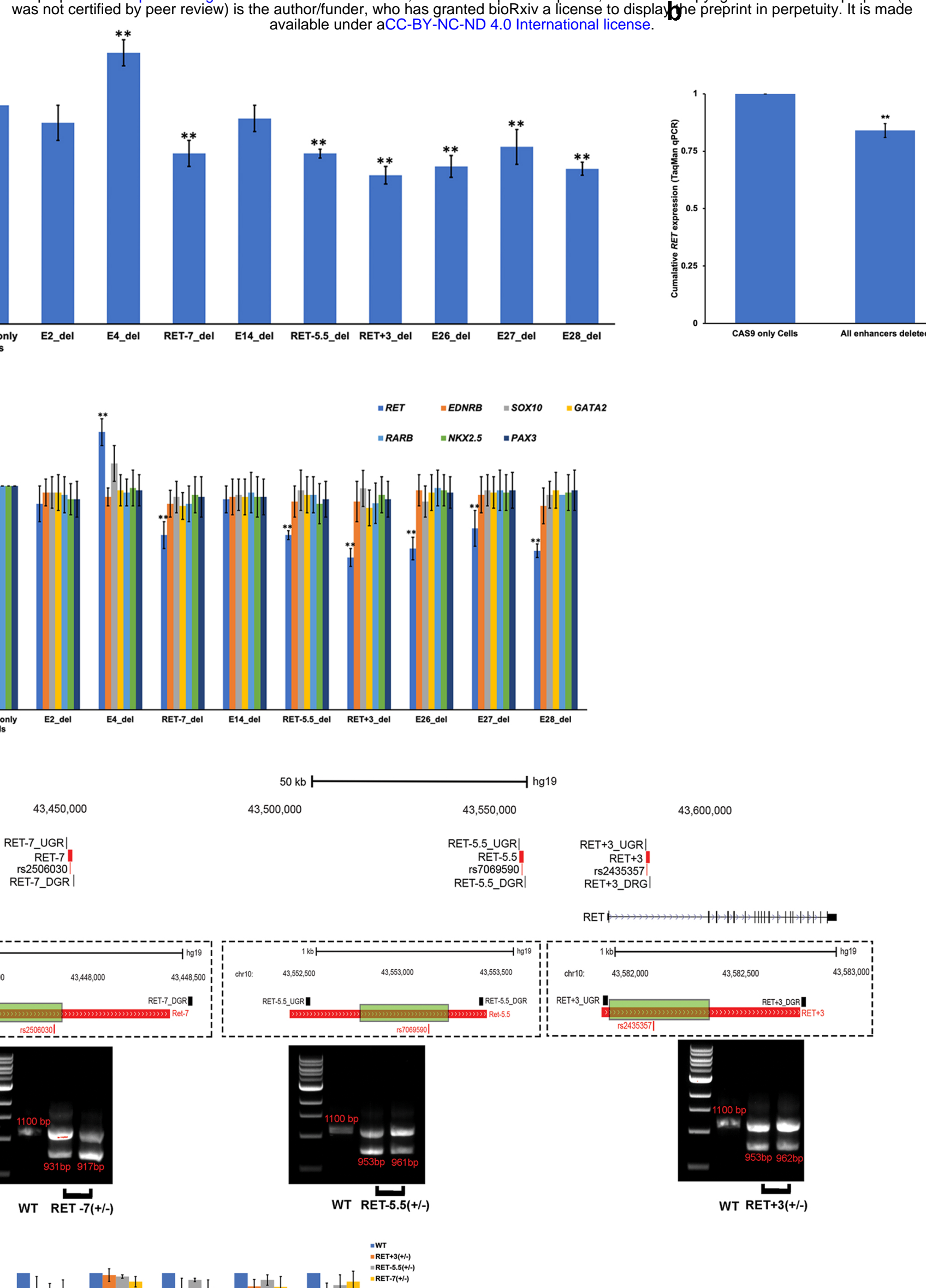

$43,550,000$

$43,600,000$

$$
\begin{array}{r}
\text { RET-7_UGR| } \\
\text { RET-7 } \\
\text { rs2506030| } \\
\text { RET-7_DGR | }
\end{array}
$$$$
\begin{array}{rr}
\text { RET-5.5_UGR| } & \text { RET+3_UGR } \\
\text { RET-5.5 } & \text { RET+3 } \\
\text { rs7069590| } & \text { rs2435357| } \\
\text { RET-5.5_DGR } & \text { RET+3_DRG }
\end{array}
$$

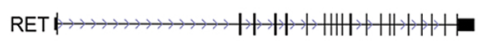

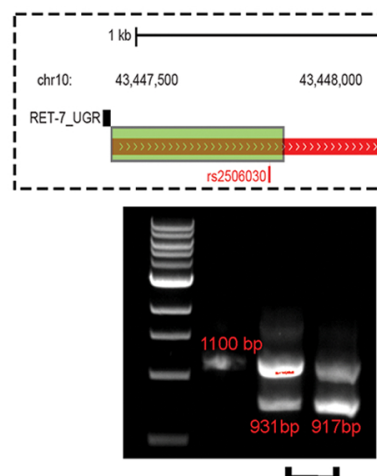

e
WT RET-5.5(+/-)
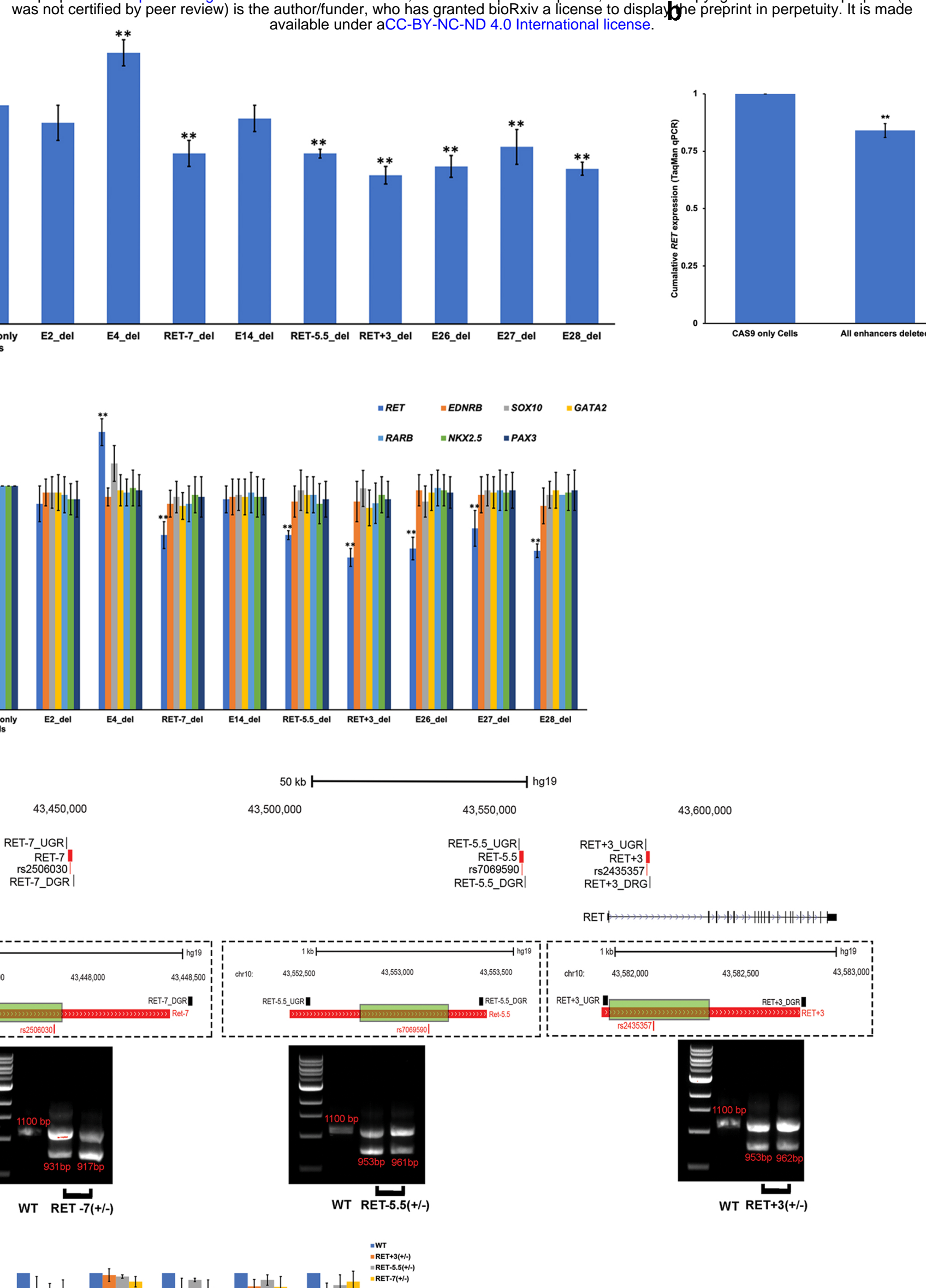

$=$ WT
$=\operatorname{RET}+3(+/-)$

$=$ RET-5.5(+l-)

$=$ RET- $7(+1-$ )

WT RET+3(+/-)
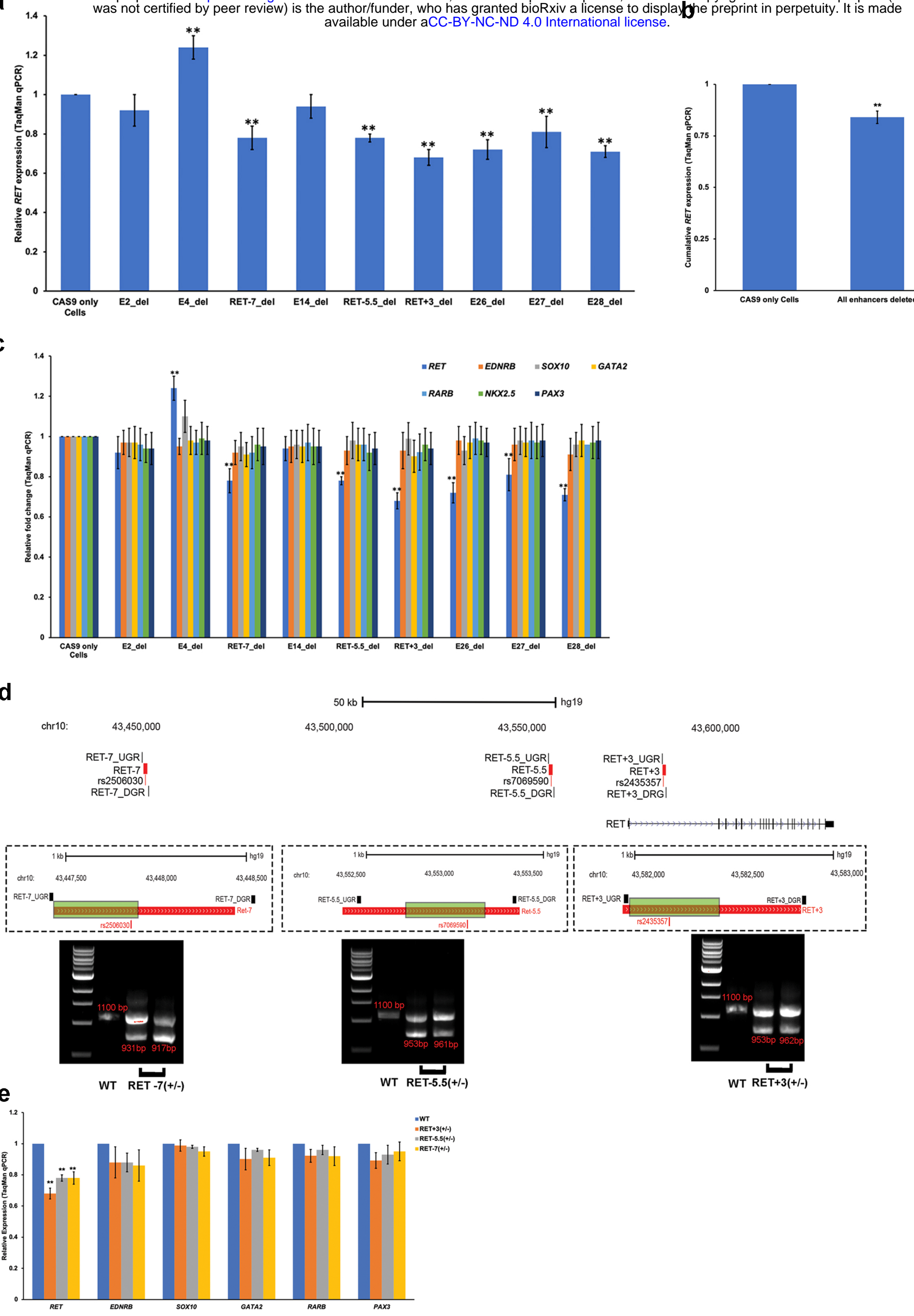